\title{
QUASARS IN THE FIELD OF SA94 - A colour survey
}

\author{
S.Cristiani (1), C.Barbieri (1), A.Iovino (2), F.La Franca (1), A.Nota (3) \\ 1) Dipartimento di Astronomia della Università di Padova \\ 2) European Southern Observatory \\ 3) Space Telescope Science Institute
}

\section{THE DATA}

In Barbieri and Cristiani (1986) the results of a preliminary objective prism survey in the SA94 were reported, in Barbieri et al. (1987) these results were compared with the colour survey of Mitchell et al. (1984). The study continued with the creation of a sample of UVX candidates in the 10 central square degrees of the field.

Two plates in each of the magnitude systems $\mathrm{U}, \mathrm{B}$ and $\mathrm{V}$ were obtained within two-weeks time (in order to minimize the effects of variability) with the $1 \mathrm{~m}$ ESO Schmidt telescope at La Silla. A two-colour diagram was derived for objects in the magnitude range $16.0<B<20.0$ with internal errors of 0.1 magnitudes both in U-B and B-V. The completeness limit of the survey has been estimated to be fainter than $B=19.8$.

The selection of the quasar candidates has been carried out using a modified Braccesi less-restricted criterium, (Braccesi et al., 1980). The follow-up observations of the quasar candidates have been carried out at the $3.6 \mathrm{~m}$ and the $2.2 \mathrm{~m}$ ESO telescopes at La Silla, equipped either with the ESO Faint Object Spectrograph and Camera (EFOSC), or with the Boller and Chivens Spectrographs, or with the OPTOPUS multi-fiber facility. Spectra have been taken for all the candidates down to $B=19.6$, while in the range $19.6<B \leq 19.8$ only 25 out of 37 candidates have been observed. 99 extragalactic emission-line objects have been confirmed.

\section{DISCUSSION AND CONCLUSIONS}

In Table 1, the surface densities for the quasars observed in the present UVX survey of SA 94 are given and compared with the data by Boyle (1987, DHM survey), Marshall et al. (1984, AB and BF surveys) and Mitchell et al. (1984, US survey) which have sampled a comparable section of the $\log N-\log S$ diagram of quasars (Fig.1). In the comparison a correction of -0.15 magnitudes has been applied to the present survey because of galatic extinction.

In general, optical quasar samples suffer a number of problems, ranging from non-objectiveness (some surveys have been carried out by eye) to incompleteness of various origins, often introducing elusive biases (see, for example, Wampler and Ponz, 1985). An especially delicate issue is the magnitude calibration, with small errors giving origin to large uncertainties in the $\log N-\log S$. 
Table 1. Quasar surface densities

\begin{tabular}{cccccc}
\hline B Magnitude & SA94 & AB & US & $\begin{array}{c}\text { DHM } \\
\text { (for b=B+0.1) }\end{array}$ & BF \\
$\leq 17.50$ & $0.4 \pm 0.2$ & $0.08 \pm 0.05$ & $0.23 \pm 0.05$ & - & - \\
$\leq 18.00$ & $0.6 \pm 0.2$ & $0.42 \pm 0.11$ & $0.49 \pm 0.19$ & - & - \\
$\leq 18.50$ & $1.3 \pm 0.4$ & - & $1.53 \pm 0.41$ & $0.7 \pm 0.3$ & - \\
$\leq 19.00$ & $3.1 \pm 0.6$ & - & - & $2.7 \pm 0.6$ & - \\
$\leq 19.50$ & $6.4 \pm 0.8$ & - & - & $7.9 \pm 1.3$ & $8.1 \pm 2.2$ \\
$\leq 19.65$ & $7.6 \pm 0.9$ & - & - & $9.8 \pm 1.4$ & $16.3 \pm 3.1$
\end{tabular}

In the present survey we have tried to minimize these limitations selecting our candidates with the best possible accuracy. Comparison with other surveys shows a good agreement in the number-counts between $B=17.0$ and $B=19.0$, while at magnitudes fainter than $B=19.0$ a deficiency of quasars is found. From our analysis we conclude that the completeness of the present sample is at least comparable to that of the other surveys, suggesting that the most plausible interpretation of the observed discrepancy is the discordance among the calibrations of the $\mathrm{B}$ magnitude scales in the different searches.

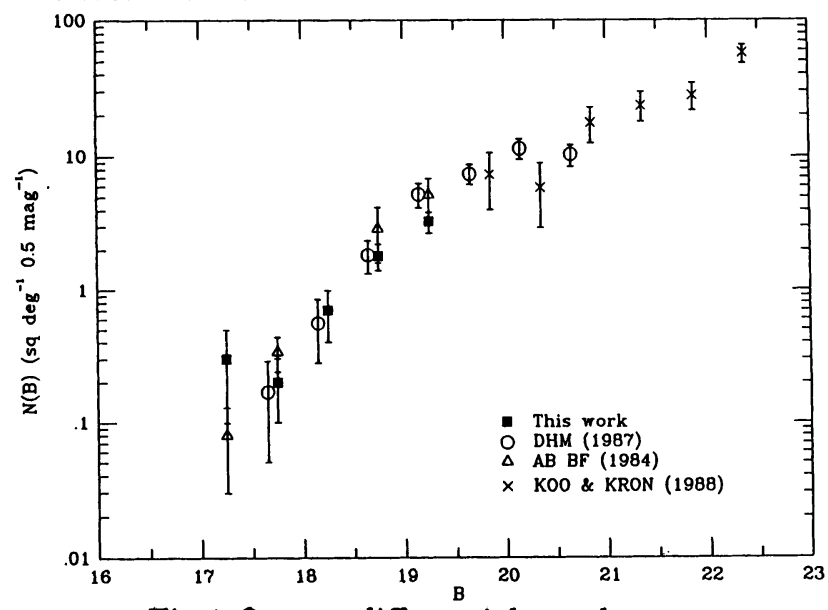

Fig.1 Quasar differential number-counts

\section{REFERENCES}

Barbieri, C., Cristiani, S., 1986, Astron. Astrophys. Suppl. 63, 1

Barbieri, C., Cristiani, S., Iovino, A., Nota, A., 1987, Astron. Astrophys. Suppl. 67,551

Boyle, B.J., Fong, R., Shanks, T., Peterson, B.A., 1987, Mon. Not. R. astr .Soc. 227, 717

Braccesi, A., Zitelli, V., Bonoli, F., Formiggini, L., 1980, Astron. Astrophys. 85, 80

Marshall, H.L., Avni, Y., Braccesi, A., Huchra, J.P., Tananbaum, H., Zamorani, G., Zitelli, V., 1984, Astrophys. J. 283, 50

Mitchell, K.J., Warnock, A., Usher, P.D., 1984, Astrophys. J. 287, L3

Wampler, E.J., Ponz, D., 1985, Astrophys. J. 298, 448 